\title{
Cluster-based routing protocols for wireless sensor networks
}

\author{
Huanan Zhang* \\ School of Data and Computer Science, Guangdong Peizheng College, Guangzhou, 510830, China
}

\begin{abstract}
Routing technology at the network layer is pivotal in the architecture of wireless sensor networks. As an active branch of routing technology, cluster-based routing protocols excel in network topology management, energy minimization, data aggregation and so on. In this paper, cluster-based routing mechanisms for wireless sensor networks are analyzed. Cluster head selection, cluster formation and data transmission are three key techniques in cluster-based routing protocols. As viewed from the three techniques, recent representative cluster-based routing protocols are presented, and their characteristics and application areas are compared. Finally, the future research issues in this area are pointed out.
\end{abstract}

\section{Introduction}

As a new information acquisition method and processing mode, Wireless Sensor Network (WSN) has become a research hotspot attracting much attention at home and abroad. WSN collects the information of the perceived objects in the network coverage area through a large number of sensor nodes deployed in the monitoring area, and provides the collected and processed information to end users through multi-hop wireless communication. WSN does not need fixed network support and is characterized by rapid deployment and strong destruction resistance. WSN can be widely used in military reconnaissance, environmental monitoring, medical monitoring, agricultural breeding and other commercial fields, as well as special fields such as space exploration and disaster rescue.

\section{Overview of WSN clustering routing protocol}

In WSN architecture, the network layer routing technology has an important impact on the performance of WSN. With the research and development of WSN, many routing protocols have been proposed. From the perspective of network topology, we can roughly divide them into two categories Planar routing protocol and cluster routing the agreement. In planar routing protocol, the status of all network nodes is equal, and there is no difference between levels and levels. They generate routing by local operations and feedback to each other. In this kind of protocol, the destination node issues a query command to the source node in the

\footnotetext{
* Corresponding author: Topwn2008@163.com
} 
monitoring area, and after the node in the monitoring area receives the query command, it sends the monitoring data to the destination node. The advantages of planar routing are that it is simple and easy to be extended without any structural maintenance work, and all network nodes have equal status and are not easy to produce bottleneck effect, so it has better robustness. The biggest disadvantages of planar routing are as follows: there is no management node in the network, lack of optimal management of communication resources, complex self-organizing collaborative working algorithm, and slow response speed to dynamic changes in the network.

In the clustering routing protocol, the network is usually divided into clusters. The cluster is a set of network nodes with some kind of correlation. Each cluster is composed of a cluster head and multiple cluster members. The cluster heads of the lower level network are the members of the cluster in the higher level network, and the cluster heads at the highest level communicate with base station BS(as shown in Fig.1). Such algorithms divide the entire network into contiguous regions.

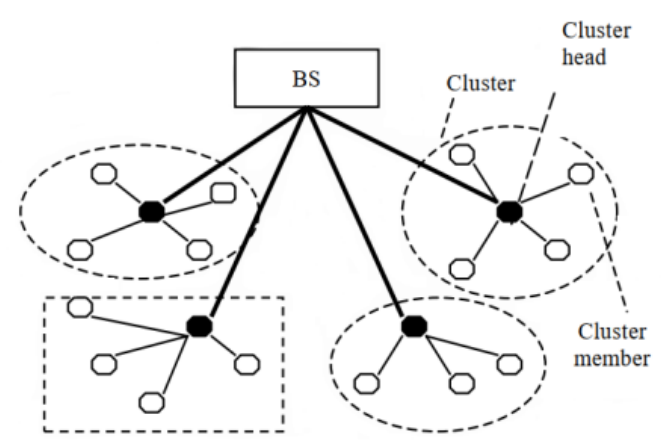

Fig. 1. Topological architecture of cluster-based routing protocols.

Under the topology management mechanism of clustering, nodes in the network can be divided into two categories: cluster-head node and member node. Within each cluster, a node is selected as the cluster head according to a certain machine algorithm, which is used to manage or control the member nodes in the entire cluster, coordinate the work among member nodes, and be responsible for the collection of information within the cluster, data fusion processing and inter-cluster forwarding.

Clustering routing mechanism has the following advantages:

(1) Member nodes can shut down the communication module most of the time, and cluster heads constitute a higher layer of connected network to be responsible for long-distance routing of data. This not only ensures the original coverage of data communication, but also saves network energy to a large extent;

(2) The cluster head fuses the data of the member nodes and then forwards the data, which reduces the traffic of data and thus saves network energy;

(3) The function of member nodes is relatively simple, and there is no need to maintain complex routing information. This greatly reduces the amount of routing control information in the network and reduces the traffic;

(4) Clustering topology is easy to manage, is conducive to the application of distributed algorithm, can respond quickly to system changes, has good scalability, and is suitable for large-scale network;

(5) Compared with planar routing, it is easier to overcome the problems caused by sensor node movement. 


\section{WSN clustering routing protocol analysis}

Since WSN features are different from Ad Hoc networks, especially WSN nodes with more limited energy, it is necessary to study a new clustering algorithm based on WSN features. LEACH(Low-Energy Adaptive Clustering Hierarchy) is the earliest clustering routing protocol proposed in WSN. Its clustering idea is embedded in many clustering routing protocols developed later, such as TEEN(Threshold sensitive Energy Efficient Sensor Network Protocol),HEED(Hybrid energy-efficient Distributed Clustering), etc. Of course, many cluster routing protocols are developed independently, such as ACE(Algorithm for Cluster Establishment),LSCP(Lightweight Sensing and Communication Protocols).

LEACH the basic idea is: through the probability and random selection of cluster, such as the energy to the entire network load evenly distributed to each sensor section point, so as to reduce the network energy consumption to extend network life cycle. The purpose of the implementation process of LEACH is cyclical, circulation, the basic process is: each round in the establishment of the cluster phase, each node to select a random number between 0 and 1 , if the number is less than a certain threshold, the node to become cluster head. Then, cluster heads to all broadcasts the news of himself as a cluster head nodes. Each node decides which cluster to join according to the strength of the broadcast signal received, and returns the cluster head. In the data transfer stage, all nodes in the cluster send data to the cluster hair according to TDMA(time division multiplexing) time slot. The cluster head will fuse the data and send the results to the base station. After a period of continuous work, the network enters the startup stage again, and the next round of cluster head selection is carried out and the cluster is rebuilt.

From the execution process of LEACH algorithm, we can find that each cycle roughly includes the following stages :(1) generation of cluster head;(2) Cluster formation;(3) Cluster routing. In fact, whether it is a clustering algorithm derived from LEACH's clustering idea or a clustering protocol independently developed, its clustering routing protocol design generally includes the above three stages.

The generation of cluster heads is the foundation of cluster formation, and the routing of cluster, namely the data transmission of cluster, depends on the structure of cluster. They are the key technologies in the design of WSN clustering routing protocol. They are closely related but relatively independent. After the cluster head is generated, different clustering strategies can be adopted, and the same cluster can also adopt different data transmission mechanisms. All WSN clustering algorithms are designed around how to select cluster heads, how to cluster and how to transmit data. Therefore, many researchers at home and abroad have proposed many excellent algorithms and protocols. Among them, some protocols have designed a complete set of algorithms from cluster head selection to data transmission, while some protocols have proposed their own algorithms for only one phase. However, no matter what phase of the protocol is, how to save energy and extend the network life cycle is the core issue that must be considered. The following is our current WSN main clustering routing protocol. From the three key technologies of clustering routing protocol design, various implementation algorithms at each stage are discussed respectively, and their application, advantages and disadvantages are analyzed, hoping to play a guiding and promoting role in future research.

\section{Cluster head generation algorithm}

The generation of cluster head is the foundation of cluster formation. The first step of clustering routing algorithm is to consider how to generate cluster heads. In some protocols, such as ECMR(Energy-Conscious Message Routing), cluster heads are pre-specified for 
deployment, assuming that their energy is not constrained. This is different from the general WSN situation. Most clustering routing protocols make resource-constrained sensor nodes assume the task of cluster head. In order to prolong the lifetime of the network, the cluster heads need to be updated periodically. The number and location of the generating methods of cluster heads determine the size and number of the final cluster structure, and also affect the energy consumption schedule of the nodes and the life cycle of the network. Current cluster-head selection algorithms are generally based on the following criteria: (1) residual energy of nodes; (2) Distance between cluster head and base station; (3) The location distribution of cluster heads, including the connectivity and coverage of cluster heads; (4) Intra-cluster communication cost.

According to the different ways of generating cluster heads, cluster heads can be divided into distributed and centralized algorithms. Distributed algorithms include two categories: one is that nodes decide whether to elect cluster heads or not according to a certain threshold value, such as LEACH; The other is to generate cluster heads dynamically through the information interaction between nodes, such as HEED. Centralized algorithm means that the cluster head is selected by the base station based on the whole network information, such as leach-C (Leach-centralized).

\section{Cluster formation algorithm}

After the cluster-head is generated, the cluster-head broadcasts the selected message to the surrounding nodes, and the surrounding nodes decide which cluster to join according to the strength of the received cluster-head broadcast signals. This is how most protocols, such as LEACH, form clusters. The implementation mechanism of this method is relatively simple, but it does not consider the size and number of clusters and load balancing from the perspective of energy. Many algorithms have proposed different clustering methods based on these problems.

Ghiasi et al. summarized the clustering algorithm as: select $\mathrm{K}$ cluster heads and divide $\mathrm{n}$ nodes into $\left(\mathrm{S}_{1}, \mathrm{~S}_{2} \ldots \mathrm{S}_{\mathrm{k}}\right) \mathrm{k}$ clusters, so that:

(1) Each node belongs to and only belongs to a cluster.

(2) Load balance between cluster heads, i.e

$$
\frac{1}{\mathrm{k}}-\gamma \leq \frac{s_{i}}{n} \leq \frac{1}{k}+\delta, i=1, \ldots k
$$

$\delta$ is the imbalance factor, which depends on the actual load capacity difference between the cluster heads. In order to uniformly consume the network energy, our goal is to pursue cluster head the load balance between, and $\delta=0$.

(3) The sum of energy consumption of clusters is the smallest, i.e

$$
c_{\mathrm{i}}=\sum_{x \subset s_{i}} f\left(x, a_{i}\right)
$$

$a_{i}$ is the cluster head of $s_{i}, x$ is the member node in the cluster, and function $f$ is the communication cost between the cluster head and the member node.

\section{Data transmission of cluster}

According to the number of hops from the member nodes to the cluster head, the structure of the cluster can be generally divided into single-hop network and multi-hop network: single-hop network such as LEACH algorithm cluster; A cluster formed by algorithms such as 
LSCP in multi-hop networks. In addition, there are other structural clusters, such as the cluster formed by PEGASIS is a chain. The routing of the cluster depends on the structure of the cluster. The structure of the cluster generated by the clustering algorithm determines the data transmission mode of the cluster to a certain extent.

ECMR(Energy-Conscious Message Routing) is a multi-hop routing protocol that assumes that the cluster head is pre-deployed, has unlimited energy, and can communicate directly with member nodes that require multi-hop routing to reach the cluster head. Therefore, the protocol considers how to establish the routing mechanism from multiple member nodes to the cluster head. This is a minimum cost path problem from the source point to the end point, and the ECMR is solved by the cluster head using Dijkstra algorithm. Among them, the definition of weights of links between nodes not only calculates the communication cost between them, but also takes into account factors such as node energy data delay and link load.

Since the energy consumption model of nodes is determined: communication energy calculation model, data processing energy calculation model and environmental perception energy calculation model. Each time a message is received, the cluster head can track the energy change of all nodes that the message passes through according to the length of the message and the amount of data and according to the energy calculation model.

In each cycle, the sensing node is only responsible for monitoring the environmental collection data; The forwarding node is only responsible for forwarding data; The perceptive forwarding node has two functions. An inactive node enters a sleep state. The cluster head determines the state of each node according to the topology of node energy and network tasks. After routing is generated, the cluster head broadcasts node state and routing information to each node. Due to the data processing and delay of message loss, the energy calculation model of the cluster head may be deviated, so the member node is required to send the energy update message directly to the cluster head regularly, report its current energy, and regenerate the optimal route from the cluster head to inform the member node in the cluster.

ECMR has good energy saving performance, high throughput and low communication delay during operation. However, this protocol has poor scalability and requires the deployment of new cluster heads to extend the network. Moreover, it relies heavily on cluster heads and does not support node movement.

\section{Summary}

In this paper, the typical clustering routing algorithms and their characteristics are systematically described from the perspective of the formation of cluster heads and routing of clusters. In our opinion, in the design of clustering routing protocol, the problem of WSN clustering routing can be further solved from the following three perspectives.(1)In cluster head selection, more effective cluster head selection algorithm and cluster head load balancing algorithm are considered. Energy is not the only constraint factor in picking cluster heads. In complex specific applications, we must also consider the distance computing power and mobility of node location and base station, especially the application that sticks Node.(2)During the formation of clusters, the control message overhead and the frequency of topology changes in the process of topology generation should be reduced to reduce the network communication overhead.(3)The design of a new data transmission mode with the advantages of both plane structure and cluster structure is an interesting research direction at present. In some cluster-based routing protocols, there is no concept of cluster-head. Each node knows the next node it will forward. We call such protocols as virtual cluster-based flat routing protocols. In our opinion, they are essentially flat routing protocols. 
In the future research, energy-aware QoS clustering routing is paid more and more attention, and it will be applied in real-time tracking of targets, which puts forward strict requirements for bandwidth guarantee and effective utilization of energy-efficient paths. In addition, the development of sensor hardware will have an important influence on the design of routing protocol. For example, the energy of ordinary sensors cannot be renewed, but advances in hardware technology make it possible to replenish the energy.

\section{References}

1. S. Madden, M. J. Franklin, J. M. Hellerstein and W.Hong, "TAG: A Tiny AGregation Service for Ad-Hoc Sensor Networks," Proceedings of the 5th Symposium on Operating Systems Design and Implementation, Boston,9-11 December 2002, pp. 131-146.

2. Y. Wu, L. X. Yang, Y. Liu and W. Lou, "Energy-Efficient Wake-Up Scheduling for Data Collection and Aggregation," IEEE Transactions on Parallel and Distributed Systems, Vol. 21, No. 2, 2010, pp. 275-287.

3. Wang, Q.; Dai, H.-N.; Cheang, C.F.; Wang, H. Link Connectivity and Coverage of Underwater Cognitive Acoustic Networks under Spectrum Constraint. Sensors 2017, 17, 2839.

4. Wang, Z.; Han, G.; Qin, H; Zhang, S.; Sui, Y. An energy-aware and void-avoidable routing protocol for underwater sensor networks. IEEE Access 2018, 6, 7792-7801.

5. Kumar, S.; Chaurasiya, V.K. A Strategy for Elimination of Data Redundancy in Internet of Things (IoT) Based Wireless Sensor Network (WSN). IEEE Syst. J. 2019, 13, $1650-1657$. 